\title{
Risk Factor Analysis of Pan-drug Resistant Acinetobacter baumannii-induced Ventilator- associated Pneumonia in ICU
}

\author{
Z. YUN ${ }^{1}$, Z. $\mathrm{HONG}^{2}$, Z. DENGCHUAN 1 , Z. AFANG ${ }^{1}$ AND L. JIABIN*
}

${ }^{1}$ Department of Emergency ICU, ${ }^{1}$ Department of Emergency, ${ }^{2}$ Department of Infectious Disease, The First Affiliated Hospital of Anhui Medical University, ${ }^{3}$ Department of Infectious Disease, The First Affiliated Hospital of Anhui Medical University, China

Zheng et al.: Drug resistant bacteria-induced ventilator-associated pneumonia

\begin{abstract}
This study was to investigate the risk factors of ventilator-associated pneumonia caused by pan-drug resistant Acinetobacter baumannii in emergency intensive care unit so as to provide related basis for clinical prevention. A total of 105 emergency intensive care unit patients, who were treated with ventilator over $48 \mathrm{~h}$ in the First Affiliated Hospital of Anhui Medical University and developed Acinetobacter baumanniiinduced ventilator-associated pneumonia from January 2017 to December 2018 were enrolled in this study, including 70 cases with pan-drug resistant Acinetobacter baumannii infection served as the pan-drug resistant group and 35 cases with non-pan-drug resistant Acinetobacter baumannii infection served as the control group. The univariate analysis and logistic regression method were used to explore the risk factors of ventilator-associated pneumonia caused by pan-drug resistant Acinetobacter baumannii. The result of univariate analysis showed that the risk factors of pan-drug resistant Acinetobacter baumannii-induced ventilator-associated pneumonia included tracheotomy, enteral nutrition, the use of carbapenems and use of more than 3 antibiotics before infection. Logistic regression analysis showed that enteral nutrition and the use of carbapenems were independent risk factors of pan-drug resistant Acinetobacter baumanniiinduced ventilator-associated pneumonia. In conclusion, enteral malnutrition and the use of carbapenems were the two significant risk factors for pan-drug resistant Acinetobacter baumannii-induced ventilatorassociated pneumonia, which are necessary to take active preventive measures in clinical practice.
\end{abstract}

Key words: Intensive care unit, pan-drug resistant Acinetobacter baumannii, ventilator-associated pneumonia, logistic regression analysis, Risk factors

Acinetobacter baumannii is widely found in water, soil, hospital environment and human skin. It is a kind of conditional pathogenic bacteria, but with the application of broad-spectrum antibiotics and glucocorticoids as well as the extensive development of invasive medical procedures, A. baumannii has become the main pathogen of nosocomial infection, especially as an important pathogen of ventilator-associated pneumonia (VAP). According to the monitoring data of nosocomial infection in the USA and domestic survey analysis on the common pathogenic bacteria of hospital infection, $A$. baumannii infection ranks fourth among all nosocomial infections. In fact, A. baumannii infection is most commonly seen in lungs and becomes an important pathogen of hospital-acquired pneumonia (HAP), especially of $\mathrm{VAP}^{[1]}$. The rate of aqcuisition drug resistances of $A$. baumannii has increased year after year with the emergence of multidrug resistant and even pan-resistant strains, which seriously threaten human health and became a major problem demanding prompt solution in clinical practices ${ }^{[2]}$. In recent years, extensive use of abtibiotics has boceme a major contributing factor for the incraesed resistance acquired by $A$. baumannii. The pan-drug resistant $A$. baumannii (PDRAB) is increasing, especially in the intensive care unit (ICU) severe burns wards ${ }^{[3]}$. PDRAB is almost resistant to all commonly used antibiotics and is easy to cause nosocomial outbreak through cross infection, which a great difficulty to treat the infection ${ }^{[4,5]}$. At present, there are few investigations on the clinical features and risk factors of PDRAB -induced VAP in ICU wards. The study was mainly aimed to perform prospective monitoring on the ICU patients treated on the ventilator over $48 \mathrm{~h}$ in the First Affiliated Hospital

*Address for correspondence

E-mail: xiachen80653@163.com

Special Issue 1, 2020 
of Anhui Medical University from January 2017 to December 2018 to explore the risk factors of PDRABinduced VAP so as to provide relevant basis for prevention of the infection. A total of 105 ICU patients, who were treated on ventilator over $48 \mathrm{~h}$ in this hospital and developed A. baumannii-induced VAP from January 2017 to December 2018 were enrolled in this study, including 70 cases with PDRAB infection served as the PDR group and 35 cases with non-PDRAB infection served as the control group. All patients participated in the study have signed the informed consent form. With combination of relevant literature and clinical practices, possible risk factors of PDRABinduced VAP were screened out followed by the development of questionnaire, the patients treated with ventilator over $48 \mathrm{~h}$ were prospectively monitored by ICU doctor, and the information of the incidence of PDRAB induced VAP was recorded followed by statistical analysis. VAP diagnosis was implemented according to diagnostic criteria for nosocomial infection issued by Ministry of Health, People's Republic of China, there is new or persistent or progressive infiltration in the chest X-ray film with more than 3 indices, which were, fever with rectal temperature was over $38^{\circ}$ or under $35^{\circ}$; white blood cell count $>10 \times 109 / 1$ with Gram's staining there were over 10 white blood cells at each high magnification; the culture of endotracheal aspiration was positive. The data were analyzed on SPSS 21 software, the count data were checked by $\mathrm{X}^{2}$ test and the measurement data $t$ test. The logistic regression was used for multivariate analysis, $\mathrm{p}<0.05$ suggested that there was difference of statistical significance. A total of 105 ICU patients were enrolled in this study, including 70 cases with PDRAB infection served as the PDR group and 35 cases with non-PDRAB infection served as the control group. There were no statistically significant differences between the 2 groups in general information such as the gender, age, APACHE II, GCS score, ventilation time, mechanical ventilation time before positive culture, mixed infection, fungal infection and smoking history, as shown in Table 1. The univariate analysis showed that the risk factors of PDRAB-induced VAP included tracheotomy, enteral nutrition, the use of carbopenems and the use of more than 3 antibiotics before infection, as shown in Table 2. The logistic regression analysis was conducted on the 4 factors of statistical significance by univariate analysis

TABLE 1: COMPARISON OF GENERAL DATA BETWEEN THE TWO GROUPS

\begin{tabular}{lcccccccccc}
\hline Group & $\mathrm{n}$ & male & Age (y) & APACHE II & GCS & $\begin{array}{c}\text { ventilation } \\
\text { time (d) }\end{array}$ & $\begin{array}{c}\text { ventilation time } \\
\text { before positive } \\
\text { culture (d) }\end{array}$ & $\begin{array}{c}\text { mixed } \\
\text { infection }\end{array}$ & $\begin{array}{c}\text { fungal } \\
\text { infection }\end{array}$ & $\begin{array}{c}\text { smoking } \\
\text { history }\end{array}$ \\
\hline $\begin{array}{l}\text { PDR } \\
\text { group }\end{array}$ & 70 & 36 & $57.5 \pm 7.5$ & $23.4 \pm 1.7$ & $9.6 \pm 1.7$ & $21.8 \pm 4.6$ & $8.2 \pm 1.2$ & 7 & 9 & 23 \\
$\begin{array}{l}\text { Control } \\
\text { group }\end{array}$ & 35 & 19 & $57.3 \pm 6.8$ & $23.2 \pm 1.9$ & $9.5 \pm 1.5$ & $21.4 \pm 3.7$ & $8.3 \pm 1.1$ & 4 & 6 & 11 \\
t/X & & 0.076 & 0.652 & 0.187 & 0.057 & 0.673 & 0.096 & 0.051 & 0.35 & 0.022 \\
$\mathrm{P}$ & $>0.05$ & $>0.05$ & $>0.05$ & $>0.05$ & $>0.05$ & $>0.05$ & $>0.05$ & $>0.05$ & $>0.05$ \\
\hline
\end{tabular}

TABLE 2: UNIVARIATE ANALYSIS ON RISK FACTORS OF PDRAB-INDUCED VAP

\begin{tabular}{|c|c|c|c|c|}
\hline Variable & PDRAB group $(n=70)$ & Control group $(n=35)$ & $\mathrm{X}^{2}$ & $\mathbf{P}$ \\
\hline \multicolumn{5}{|l|}{ Associated disease } \\
\hline Diabetes & 7 & 4 & 0.051 & $>0.05$ \\
\hline Cardiovascular diseases & 15 & 8 & 0.028 & $>0.05$ \\
\hline Lung diseases & 16 & 9 & 0.015 & $>0.05$ \\
\hline \multicolumn{5}{|l|}{ Invasive operation } \\
\hline Central venous catheterization & 57 & 29 & 0.032 & $>0.05$ \\
\hline Catheter & 64 & 31 & 0.221 & $>0.05$ \\
\hline Racheotomy & 40 & 11 & 6.176 & $<0.05$ \\
\hline Enteral nutrition & 56 & 16 & 12.727 & $<0.05$ \\
\hline Hormone use & 41 & 20 & 0.02 & $>0.05$ \\
\hline Antacid use & 64 & 31 & 0.221 & $>0.05$ \\
\hline \multicolumn{5}{|l|}{ Antibiotic use } \\
\hline Piperacillin-tazobactam & 34 & 16 & 0.076 & $>0.05$ \\
\hline Third-generation cephalosporin & 33 & 16 & 0.019 & $>0.05$ \\
\hline carbapenems & 36 & 7 & 9.531 & $<0.05$ \\
\hline Use of more than 3 antibiotics before infection & 43 & 10 & 10.077 & $<0.05$ \\
\hline
\end{tabular}


and the results showed that enteral nutrition as well as the the use of carbapenems were independent risk factors of PDRAB-induced VAP, as shown in Table 3. $\mathrm{VAP}$, one of HAP, refers to the pneumonia occurring since $48 \mathrm{~h}$ after mechanical ventilation. It is a common and serious complication in the process of mechanical ventilation. Once the patients have VAP, they are prone to difficulty of extubation, thereby prolonging the duration of hospitalization with increased expenses and even threatening the patients to death. Due to the specialty in pathogenic bacteria, diagnosis, treatment and higher mortality, VAP has attracted widespread attention in recent years. It has certain local and epidemiological characteristics and its occurrence is closely related to potential diseases, past use of antibiotics, transmission routes and source of pathogenic bacteria. Pathogenic spectrum varies from region to region ${ }^{[6,7]} .90 \%$ of the pathogenic bacteria were bacterial infections, and the infection rate of Gram-negative bacteria was higher. Generally, old patients have more severe underlying disease, low resistance and long hospital stay, and some patients may have lower immunity due to the use of immunosuppressants and hormones ${ }^{[8,9]}$. Invasive procedures like endotracheal intubation make damages to local defense mechanism, leading to the condition that the pathogens in the upper respiratory tract are prone to spreading to the lower respiratory tract. In addition, the wide application of broad-spectrum antibiotics also leads to elevated infection rate of resistant opportunistic pathogens, flora imbalance and increased fungal infection ${ }^{[10]}$. The therapy of VAP included, treatment of primary diseases, prevention of risk factors, nutritional support and immunological-support treatment along with application of reasonable antibiotics, the most important in this regard. At early stage, the correct use of antibiotics can reduce the mortality in patients. Because the diagnosis of VAP is difficult, patients highly suspected with VAP can be timely treated with empirical antibiotics simultaneously with bacterial cultures and drug sensitivity test. With the transition of pathogenic bacteria and the emergence of multiple antibiotic resistant strains, the choice of antibiotics for VAP treatment is also changing ${ }^{[11]}$. A. baumannii has become an important clinical pathogen and is characterized by fast obtaining, resistance spreading, multi and widespread-drug resistance. PDRAB has been worldwide epidemic and became a global challenge in the field of antiinfective therapy ${ }^{[12]}$. MDRAB is defined to be resistant to at least 3 of such agents as cephalosporins against Pseudomonas, carbapenem antibiotics against Pseudomonas, complex preparation containing 13-lactamase inhibitors, fluoroquinolones and aminoglycosides ${ }^{[13]}$. Extensively-drug resistant A. baumannii refers to the strains sensitive to only 1 or 2 types with potential anti acinetobacter activity, commonly including tigecycline and colistin. A. baumannii infection is more commonly seen in critically ill patients and often accompanied by other bacterial infections or fungal infections, leading to high mortality in patients ${ }^{[14]}$. In this study, the comparison of subjects' general data between the PDR group and the control group showed no statistically significant difference in gender, age, APACHE II, GCS score, ventilation time, mechanical ventilation time before positive culture, mixed infection, fungal infection and smoking history, suggesting that there is no obvious difference in general epidemiological characteristics between PDRAB-induced VAP patients and non PDRAB induced VAP patients. According to the survey, PDRAB-induced VAP is related to tracheotomy and enteral nutrition with the latter being an independent risk factor. It is possibly because these invasive procedures destroy human normal defense mechanisms and therefore increase the chance of bacterial infection. When the enteral nutrition is retained in gastric tube, the stomach shows reflex inhibition with a delay of gastric emptying, likely to cause mis-inhalation and increase the chance of pulmonary infection. Besides, operation error, inadequate aseptic technique and unqualified sterilization in the process will also increase the incidence of infection in patients. The survey also showed that the number of antibiotics used before infection in the two groups was statistically different, indicating that the choice of multiple antibiotics may be an important factor of PDRAB infection. A related study has also shown that the infection of $A$. baumannii is related to the use of carbapenem antibiotics ${ }^{[15]}$. And it is also shown in this study that carbapenems is an independent risk factor for PDRAB-induced VAP, and

TABLE 3: LOGISTIC REGRESSION ANALYSIS ON RISK FACTORS OF PDRAB-INDUCED VAP.

\begin{tabular}{|c|c|c|c|c|c|}
\hline Variable & $\bar{B}$ & SE & Wald $X^{2}$ & OR $(95 \% \mathrm{Cl})$ & $\mathbf{P}$ \\
\hline Tracheotomy (with vs. without) & 0.764 & 0.503 & 1.292 & $2.044(0.692-5.738)$ & $>0.05$ \\
\hline Enteral nutrition (with vs. without) & 1.185 & 0.517 & 4.324 & $3.155(1.093-8.349)$ & $<0.05$ \\
\hline Carbapenems (with vs. without) & 1.361 & 0.374 & 5.125 & $4.326(1.254-14.316)$ & $<0.05$ \\
\hline Use of more than 3 antibiotics before infection (yes vs. no) & 0.793 & 0.684 & 1.602 & $0.571(0.168-1.372)$ & $>0.05$ \\
\hline
\end{tabular}


the resistance mechanism of $A$. baumannii against carbapenems is mainly the production of KPC-2 and blaOXA-23 type enzyme as well as absent expression of $25 \mathrm{ku}$ outer membrane protein with efflux system overexpression probably involved in drug resistance of meropenem. It is realized that following targeted prevention and control measures should be taken according to the clinical characteristics of VAP caused by PDRAB, (1) pay attention to the monitoring of PDR bacteria and implement PDRAB screening system. High-risk patients are given close monitoring with high focus on ICU patients undergoing invasive treatments such as tracheotomy, ventilator and long-term medication of antibiotics, and patients should be immediately treated in isolation as soon as they are found the signs of PDRAB infection; (2) strengthen training of knowledge about PDR bacteria infections and enhance management consciousness of the infection in medical personnel; (3) strictly carry out disinfection and isolation system as well as hand hygiene management. The sign of contact isolation is pasted on medical records and bedside the patients infected with PDRAB; the staff contacting such patients are strictly given the implement of standard precautions and hand hygiene system; when taking invasive procedures, the operating rules and systems must be strictly abided by; (4) conduct regular evaluation on the patients undergoing invasive operation to reduce invasive time and (5) make sure the correctness and timeliness of sample collection. Antibiotics are correctly chosen according to the results of drug sensitivity to prevent the resistance caused by abuse of antibiotics. Enteral malnutrition and the use of carbapenems are two significant risk factors for PDRAB-induced VAP, which are necessary to take active preventive measures in clinical practice.

\section{Conflict of interest:}

No conflict of interest between any of the authors.

\section{REFERENCES}

1. Qiu H, Zhou JY. Clinical distribution and drug resistance of 4 non-fermenting bacteria of lower respiratory tract infection. Chin J Microecol 2011;23:447-9.

2. Afkhamzadeh AR, Lahoorpour F, Delpisheh A, Janmardi R. Incidence of ventilator-associated pneumonia (VAP) and bacterial resistance pattern in adult patients hospitalised at the intensive care unit of Besat Hospital in Sanandaj. SJKU 2011;16:20-26.

3. Buckley MS, Dzierba AL, Smithburger PL, McAllen KJ, Jordan CJ, Kane-Gill SL. Chlorhexidine for the prevention of ventilator associated pneumonia in critically ill adults. J Infect Prev 2013;14:162-9.
4. Ning BT, Zhang CM, Liu T, Ye S, Yang ZH, Chen ZJ. Pathogenic analysis of sputum from ventilator-associated pneumonia in a pediatric intensive care unit. Exp Ther Med 2013;5:367-371.

5. Kalil AC, Metersky ML, Klompas M, Muscedere J, Sweeney DA, Palmer LB, et al. Management of adults with hospitalacquired and ventilator-associated pneumonia: clinical practice guidelines by the Infectious Diseases Society of America and the American Thoracic Society. Clin Infect Dis 2016;63:e61-e111.

6. He H, Zheng Y, Sun B, Tang X, Wang R, Tong Z. Tigecycline combination for ventilator-associated pneumonia caused by extensive drug-resistant Acinetobacter baumannii. J Thorac Dis 2016;8(10):2784-92.

7. Schafer JJ, Goff DA, Stevenson KB, Mangino JE. Early experience with tigecycline for ventilator-associated pneumonia and bacteremia caused by multidrug-resistant Acinetobacter baumannii. Pharmacotherapy 2007;27:980-7.

8. Yuan Z, Tam VH. Polymyxin B. Polymyxin B: A new strategy for multidrug resistant Gram-negative organism. Expert Opin Investig Drugs 2008;17:661-8.

9. Lin CC, Liu TC, Kuo CF, Liu CP, Lee CM. Aerosolized colistin for the treatment of multidrug-resistant Acinetobacter baumannii pneumonia: experience in a tertiary care hospital in northern Taiwan. J Microbiol Immunol Infect 2010;43:323-31.

10. Wang D. Experience with extended-infusion meropenem in the management of ventilator-associated pneumonia due to multidrug-resistant Acinetobacter baumannii. Int J Antimicrob Agents 2009;33:290-1.

11. Ebenezer K, James EJ, Michael JS, Kang G, Verghese VP. Ventilator-associated Acinetobacter baumannii, pneumonia. Indian Pediatr 2011;48:964-6.

12. Royer S, Faria AL, Seki LM, Chagas TP, Campos PA, Batistão $\mathrm{DW}$, et al. Spread of multidrug-resistant Acinetobacter baumannii and Pseudomonas aeruginosa clones in patients with ventilator-associated pneumonia in an adult intensive care unit at a university hospital. Braz J Infect Dis 2015;19:350-7.

13. Shin JA, Chang YS, Kim HJ, Kim SK, Chang J, Ahn CM, et al. Clinical outcomes of tigecycline in the treatment of multidrugresistant Acinetobacter baumannii infection. Yonsei Med J 2012;53:974-84.

14. Myrianthefs P, Ioannides K, Gavala A, Evodia E, Tsakris A, Baltopoulos G. Patterns of resolution of infectious parameters in multidrug-resistant Acinetobacter baumannii ventilatorassociated pneumonia. J Hosp Infect 2010;75:79-80.

15. Tsioutis C, Kritsotakis EI, Karageorgos SA, Stratakou S, Psarologakis C, Kokkini S, et al. Clinical epidemiology, treatment and prognostic factors of extensively drug-resistant Acinetobacter baumannii ventilator-associated pneumonia in critically ill patients. Int J Antimicrob Agents 2016;48:492-7.

This is an open access article distributed under the terms of the Creative Commons Attribution-NonCommercial-ShareAlike 3.0 License, which allows others to remix, tweak, and build upon the work non-commercially, as long as the author is credited and the new creations are licensed under the identical terms

This article was originally published in a special issue, "XXXXXX"

Indian J Pharm Sci 2020:82(1)spl issue1; XX-XX 